\title{
Analysis of features of recombination mechanisms in silicon solar cells
}

\author{
R.M. Korkishko, V.P. Kostylyov, N.A. Prima, A.V. Sachenko, O.A. Serba, T.V. Slusar ${ }^{1}$, V.V. Chernenko \\ V. Lashkaryov Institute of Semiconductor Physics, NAS of Ukraine, \\ 41, prospect Nauky, Kyiv 03028, Ukraine, \\ phone/fax: +38(044) 525-5788,E-mail:vkost@isp.kiev.ua \\ ${ }^{1}$ National Aviation University, 1, prospect Komarova, Kyiv 03058, Ukraine
}

\begin{abstract}
Investigated in this paper are theoretical and experimental spectral dependences of the short-circuit current as well as small-signal photo-e.m.f. in silicon solar cells. The authors have considered two constructions of solar cells. The first construction is a solar cell with contacts on the front and back surfaces, and the second solar cells with back barriers and contact metallization. Analyzed in the work are spectral dependences of the internal quantum efficiency for the short-circuit current and smallsignal photo-e.m.f.

It has been shown that the short-wave drop of the short-circuit current is related with recombination on deep centers at the front surface as well as inter-band Auger recombination in the heavily doped emitter. At the same time, availability of the shortwave drop in the small-signal photo-e.m.f. is related with limitation of the efficient rate of surface recombination $S_{\text {eff }}(\lambda)$ due to diffusion inflow.

The latter takes place when a layer with the thickness $d_{p}$ and increased recombination is available near illuminated surface. In this case, the mechanism providing decrease in the small-signal photo-e.m.f. in the area of strong light absorption is related with increasing the efficient rate of surface recombination near the front surface, when the dominant amount of electro-hole pairs is generated in the layer with the increased recombination rate. The same mechanism is responsible for the short-circuit current drop in solar cells with back barriers and contact metallization.

Juxtaposition of theoretical and experimental results enabled to determine parameters that characterize sub-surface properties of solar cells, namely: the thickness of the surface layer with increased recombination, lifetime of carriers in it, and dependences $S_{\text {eff }}(\lambda)$.
\end{abstract}

Keywords: silicon solar cell, photo-e.m.f., surface recombination.

Manuscript received 26.11.13; revised version received 00.00.13; accepted for publication 20.03.13; published online 31.03.13.

\section{Introduction}

Spectral dependences of the small-signal photo-e.m.f. and short-circuit current serves usually to determine the diffusion length of minority carriers $L$ in semiconductor or semiconductor structure, in particular, in the base of solar cells [1].

First, it should be noted that on the assumption when $L \geq d$, where $d$ is the thickness of a semiconductor sample, determination of the value $L$ is considerably complicated. Analyzed in the works [2, 3] were the possibilities to determine $L$ from spectral dependences for the short-circuit current and small-signal photo-e.m.f. in the cases when $L \geq d$, however, in these analyses possible availability of a layer with very low values of the bulk lifetimes in a narrow sub-surface area of semiconductor or semiconductor structure was not taken into account. In particular, this layer can occur due to creation of a heavily doped emitter in the solar cell. Here, inter-band Auger recombination becomes the main 
recombination mechanism in this layer, and lifetimes of non-equilibrium charge carriers are considerably reduced as compared with the lifetimes in the base. Due to it, there arises a part of the short-wave drop in the spectral dependences of the internal quantum efficiency for the short-circuit current $q(\lambda)$. This part is the clearer pronounced, the higher is the level of emitter doping and its thickness. If one uses the known method, when the spectral dependences for the short-circuit current obtained for the similar amount of incident quanta are normalized by the value at the peak, then it is easy to convenience oneself that the stronger is pronounced the drop part, the higher is the error in $L$-value determination. It should be noted that it is valid for the case when the inequality $L<d$ is rather strong, too.

It seems topical to consider the question of the possibility to determine the efficient rate of surface recombination on the illuminated surface $S_{0}$ from the drop value in spectral dependences of the short-circuit current within the short-wave range. The matter is that the influence of Auger recombination in emitter and surface recombination on the illuminated surface $S_{0}$, under some conditions, can cause practically the same decrease of $q(\lambda)$ within the short-wave absorption range. A difference between them occurs only when the efficient rate of surface recombination is close to $S_{0} \geq 10^{6} \mathrm{~cm} / \mathrm{s}$. Then, the contribution of $S_{0}$ to the shortwave drop of $q(\lambda)$ is saturated, and the increase in the doping level of the emitter and its thickness results in shifting the $q\left(\lambda_{m}\right)$ peak into the long-wave range.

\section{Spectral dependences for the short-circuit current}

When recombination on the front and back surfaces of a solar element is available, the expression for the internal quantum efficiency of the short-circuit current $q(\lambda)$ is essentially complicated. The sufficiently general expression for the $q$ value is given, for example, in [4]. It has the following look: where $\alpha(\lambda)$ is the coefficient of light absorption, $\lambda \quad-\quad$ wavelength in nanometers, $L_{p}=\left(D_{p} / C_{p} N_{d}^{2}\right)^{1 / 2}=\left(D_{p} \tau_{p}\right)^{1 / 2}-$ diffusion length for carriers in the emitter, $N_{a}$ and $D_{p}$ are doping level and diffusion coefficient for holes in the emitter, $d_{p}$ is the emitter thickness, $L$ and $D$ are the length and diffusion coefficient for minority carriers in the base, $d$ is the thickness of the solar cell, $R_{d}$ - coefficient of reflection from the back surface, $S_{0}$ - rate of recombination on the illuminated surface, $S_{d}$ - rate of recombination on the back surface.

In the case when $L<d$, the spectral dependence of internal quantum efficiency for the short-circuit current is defined by the Gertner formula

$$
q(\lambda)=\frac{\alpha(\lambda) L}{1+\alpha(\lambda) L}
$$

Depicted in Figs 1 and 2 are theoretical spectral dependences of internal quantum efficiency for the short-circuit current for silicon solar cells $q(\lambda)$.

Here, we used the following parameters: $N_{a}=10^{18}$ $(1,2,3), 6 \cdot 10^{19} \mathrm{~cm}^{-3}(4,5) ; S_{0}=1(1,2,4,5), 10^{5} \mathrm{~cm} / \mathrm{s}$ (3); $R_{d}=0(2,3,5), 0.8(1,4) ; S_{d}=1(1,2,3), 10^{5} \mathrm{~cm} / \mathrm{s}$ $(4,5)$. The curve 6 has been plotted using the Gertner formula (4).

When plotting the curves in Figs $1 \mathrm{a}$ and $1 \mathrm{~b}$, we used the diffusion length value $L=100 \mu \mathrm{m}$, while in Figs $2 \mathrm{a}$ and $2 \mathrm{~b}-L=400 \mu \mathrm{m}$. The depth of $p$ - $n$ junction location, i.e., the emitter thickness, was assumed to be $1 \mu \mathrm{m}$. Figs a and $\mathrm{b}$ differ by that the panel a shows the $q(\lambda)$ dependences within the range 400 to $1200 \mathrm{~nm}$, and the panel $\mathrm{b}$ - from 900 to $950 \mathrm{~nm}$. In these figures, for parameters of inter-band Auger recombination we used the values and relations adduced in the work [4].

Let us analyze data of Fig. 1a. When the doping level in the emitter is close to $1 \cdot 10^{18} \mathrm{~cm}^{-3}$ (curves 1 and 2) and under conditions of rather low rates of surface

$q(\lambda)=q_{p}(\lambda)+q_{n}(\lambda)$

$$
\begin{aligned}
& q_{p}(\lambda)=\frac{\alpha L_{p}}{\left(\alpha L_{p}\right)^{2}-1} \cdot \frac{\alpha L_{p}+S_{0} \frac{\tau_{p}}{L_{p}}\left(1-e^{-\alpha d_{p}}\right) \cosh \left(\frac{d_{p}}{L_{p}}\right)-e^{-\alpha d_{p}} \sinh \left(\frac{d_{p}}{L_{p}}\right)-\alpha L_{p} e^{-\alpha d_{p}}}{S_{0} \frac{\tau_{p}}{L_{p}} \sinh \left(\frac{d_{p}}{L_{p}}\right)+\cosh \left(\frac{d_{p}}{L_{p}}\right)}, \\
& q_{n}(\lambda)=\frac{\alpha L e^{-\alpha d_{p}}}{1-(\alpha L)^{2}} \cdot\left\{\frac{\left[S_{d} \cosh \left(\frac{d}{L}\right)+\frac{D}{L} \sinh \left(\frac{d}{L}\right)\right]\left(1+R_{d} e^{-2 \alpha d}\right)+\left(\alpha D\left(1-R_{d}\right)-S_{d}\left(1+R_{d}\right)\right) e^{-\alpha d}}{S_{d} \sinh \left(\frac{d}{L}\right)+\frac{D}{L} \cosh \left(\frac{d}{L}\right)}-\right. \\
& \left.\alpha L S_{d}\left[\sinh \left(\frac{d}{L}\right)+\frac{D}{L} \cosh \left(\frac{d}{L}\right)\right]\left(1-R_{d} e^{-2 \alpha d}\right)\right] \\
& \left.-\frac{S_{d} \sinh \left(\frac{d}{L}\right)+\frac{D}{L} \cosh \left(\frac{d}{L}\right)}{L}\right\}
\end{aligned}
$$


recombination, the short-wave drop is practically absent in the theoretical dependence. Its availability in the curve 3 is related with a high $S_{0}$ value. The presence of the short-wave drop in the curves 4 and 5 is related with heavy doping the emitter and a high rate of Auger recombination. As can be seen from comparison of the curves 3 and 4 , for definite parameters the spectral dependences $q(\lambda)$ within the range of the short-wave drop can be sufficiently close both for the case when only surface recombination $S_{0}$ acts on the illuminated surface, and for the case when recombination is mainly caused by Auger processes in the heavily doped emitter layer. For different curves, the $R_{d}$ value is assumed to be 0.8 or 0 . Good coincidence with the Gertner curve (4) is reached in the case $R_{d}=0$. But for $R_{d}=0.8$, it is not reached. Fig. 1b shows the spectral dependences of the internal quantum efficiency for the short-circuit current in the narrowed spectral range: 900 to $950 \mathrm{~nm}$. It is clearer seen from this figure that coincidence with the Gertner curve is only reached when $R_{d}=0$. The same fact is confirmed by the curves in coordinates $k^{-1}\left(\alpha^{-1}\right)$ plotted in Fig. 3 a.
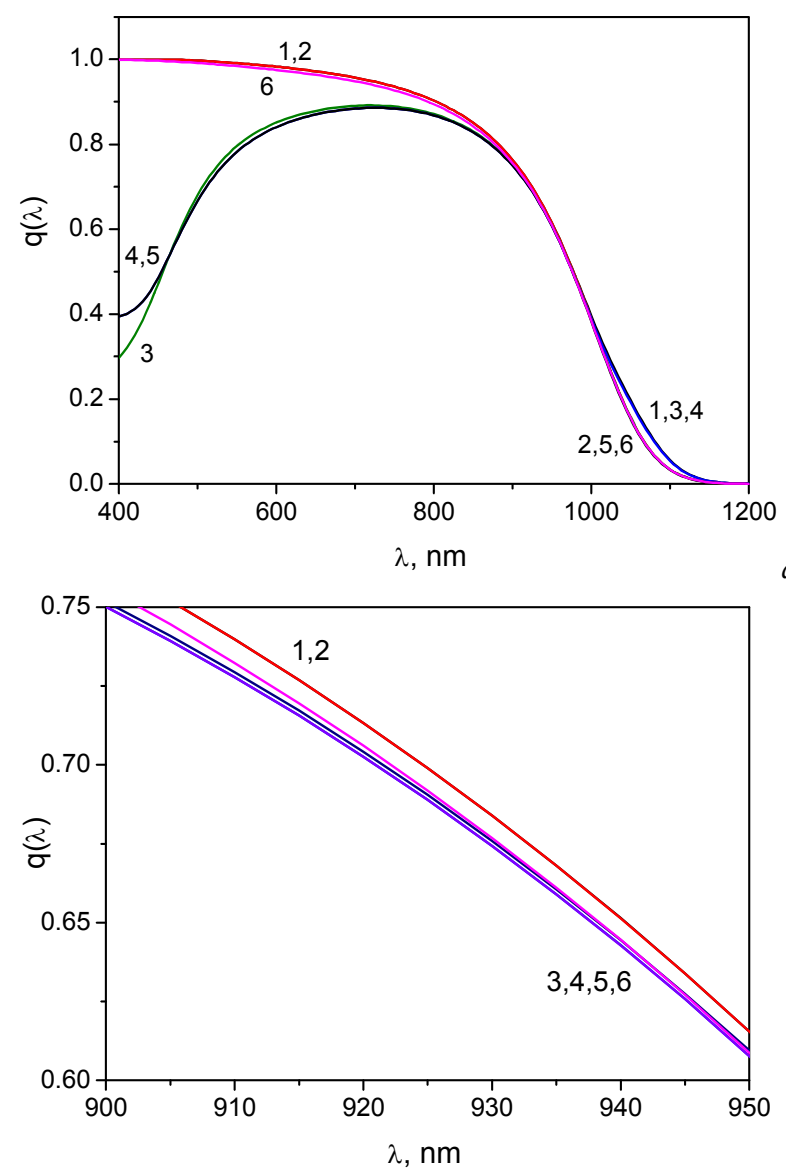

Fig. 1. Spectral dependences of the internal quantum efficiency for the short-circuit current in silicon solar cells $q(\lambda)$ in the case when the diffusion length in the base is equal to $L=100 \mu \mathrm{m}$.
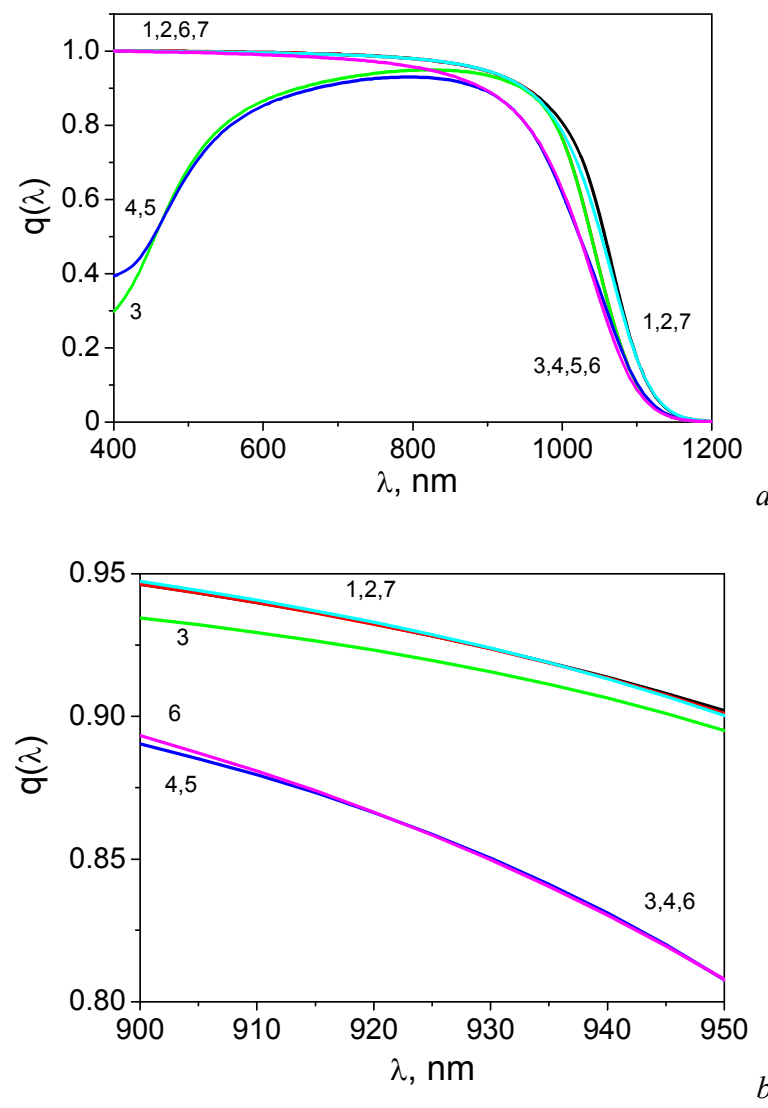

Fig. 2. Spectral dependences of the internal quantum efficiency for the short-circuit current in silicon solar cells $q(\lambda)$ in the case when the diffusion length in the base is equal to $L=400 \mu \mathrm{m}$. When plotting the curves 1 to 5 , we used the same parameters as in Fig. 1. The curves 6 and 7 have been plotted using the Gertner formula (4).

In what follows, let us consider Fig. 2a plotted for $L=400 \mu \mathrm{m}$. When the doping level in the emitter reaches $1 \cdot 10^{18} \mathrm{~cm}^{-3}$ (curves 1 and 2) and for low rates of surface recombination, the short-wave drop is not practically observed in the theoretical dependence. Its availability in the curve 3 is related with a high $S_{0}$ value. In the curves 4 to 6 , the presence of the short-wave drop is related with heavy doping the emitter $\left(\sim 6 \cdot 10^{19} \mathrm{~cm}^{-3}\right)$ as well as a high rate of Auger recombination that takes place in these conditions. When plotting the curve 6 , we took into account a high rate of recombination on the back surface $S_{d}$, therefore these dependences decay earlier in the long-wave range. The curves 6 and 7 are plotted using the Gertner formula. As can be seen from the figure, the theoretical dependences within the longwave range are approximately fitted for the values of the diffusion length in the base 580 and $270 \mu \mathrm{m}$. The first value was obtained for a low value of $S_{d}$, while the second one - for a high value. Thus, in this case fitting with the Gertner formula essentially depends on the $S_{d}$ value, and high $S_{d}$ values correspond to lower $L$ values, while at low $S_{d}$ values $L$ grows. 
From Fig. 2b, where the same spectral dependences $q(\lambda)$ are plotted in the narrowed range 900 to $950 \mathrm{~nm}$, it is well seen that fitting with the Gertner formula for high diffusion lengths, first, becomes inaccurate even in this narrow spectral range, second, for low rates of recombination on the back surface the $L$ value becomes strongly overestimated, while for high $S_{d}$ values strongly underestimated.

Plotted in Fig. 3 are dependences of the reverse internal quantum efficiency for the short-circuit current on the reverse absorption coefficient in the case when $L=100 \mu \mathrm{m}$. Also, it is clear that the theoretical dependence agrees well with that obtained using the Gertner formula only when $R_{d}=0$.

Shown in Fig. 4 are experimental and theoretical dependences $q(\lambda)$ for two high-quality silicon solar cells. Fitting the experimental and theoretical curves enabled to determine the diffusion length in the base, which was identical for both samples and equal to $450 \mu \mathrm{m}$.

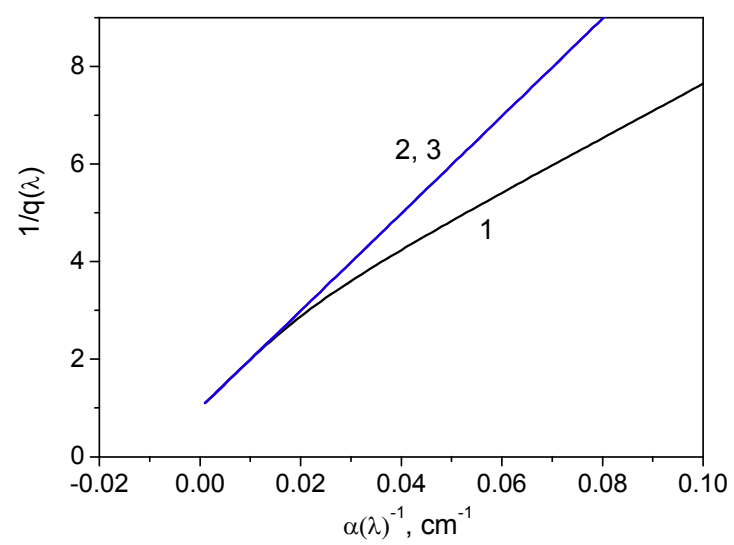

Fig. 3. Dependences of the reverse internal quantum efficiency for the short-circuit current on the reverse absorption coefficient in the case when the diffusion length in the base $L=100 \mu \mathrm{m}$. Here, we used the following parameters: curve $1-$ $N_{a}=10^{18} \mathrm{~cm}^{-3}, R_{d}=0.8$; curve $2-N_{a}=10^{18} \mathrm{~cm}^{-3}, R_{d}=0$; curve 3 is plotted using the Gertner formula.

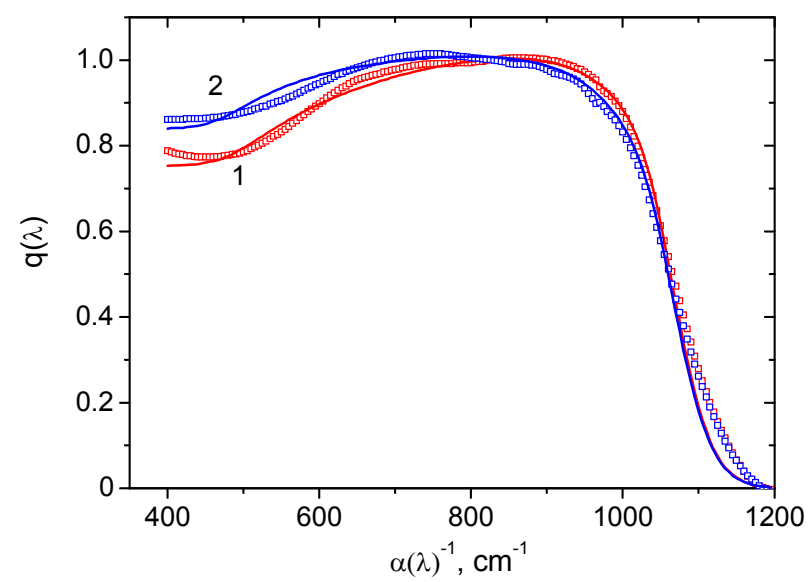

Fig. 4. Experimental and theoretical dependences $q(\lambda)$ for two high-quality silicon solar cells with a diffusion $p$ - $n$-junction.
The same fitting values were also obtained for the emitter thickness $(3 \mu \mathrm{m})$, for $R_{d}(0.8), S_{0}$ and $S_{d}$ $10^{2} \mathrm{~cm} / \mathrm{s}$. Their doping levels differ a little between one another: $10^{19} \mathrm{~cm}^{-3}$ in the first sample and $7 \cdot 10^{18} \mathrm{~cm}^{-3}-$ in the second one.

\section{Spectral dependences of the small-signal photo-e.m.f.}

Except the case of a solar cell, sub-surface layers with low lifetimes (as compared with the bulk ones) can occur in semiconductor wafers implanted with impurities that create deep bulk levels, in the case of damages that arise during mechanical processing the semiconductor samples, and so on. In all these cases, the short-wave drop appears in spectral dependences of the small-signal photo-e.m.f., too.

At last, as it was noted in $[5,6]$ the short-wave drop in the small-signal photo-e.m.f. $V_{p h}(\lambda)$ can take place as a consequence of the disturbance in constancy of the Fermi quasi-level for minority carriers or majority carriers in the sub-surface space charge region (SCR). This disturbance arises as a result of high values of the rate of surface recombination and can occur in the case when exhausting band bends take place at the surface for minority carriers.

Our investigations have shown that, if concerning the small-signal surface photo-e.m.f., for the short-wave drop to be available, it is necessary for the value of the efficient surface recombination speed on the illuminated surface $S_{0 \text { eff }}$ to be a function of the wavelength inherent to solar illumination $\lambda$, when $\lambda$ being decreased, it should grow. In particular, the following expression corresponds to this dependence:

$S_{0 e f f}(\lambda)=\left(S_{1}^{-1}+S_{2}{ }^{-1} \exp \left(-\alpha(\lambda) d_{p}\right)\right)^{-1}$.

For the short-wave drop to be real, it is necessary for the relationship $S_{1} \geq S_{2}$ to be valid. In (5), the value $d_{p}$ has the physical sense of the thickness of the layer, in which the bulk lifetime is lower than that in the base.

It is obvious from the physical viewpoint that the above dependences $S_{0 \text { eff }}$ take place when surface recombination on the illuminated side for arbitrary $\lambda$ values is limited by the speed of diffusion bringing the minority charge carriers to the surface. In particular, in the case when the layer with the thickness $d_{p}$ exists near the surface where the lifetime of minority charge carriers and their mobility are less than corresponding values in the base, then, in accord with [7], the efficient speed of surface recombination for $\alpha d_{p}<1$ is expressed as follows:

$$
S_{e f f}=\frac{D_{1}}{L_{1}} \frac{S_{0} \frac{L_{1}}{D_{1}} \cosh \left(\frac{d_{P}}{L_{1}}\right)+\sinh \left(\frac{d_{p}}{L_{1}}\right)}{S_{0} \frac{L_{1}}{D_{1}} \sinh \left(\frac{d_{p}}{L_{1}}\right)+\cosh \left(\frac{d_{p}}{L_{1}}\right)},
$$

where $D_{1}$ and $L_{1}$ are diffusion coefficient and diffusion length, respectively. 


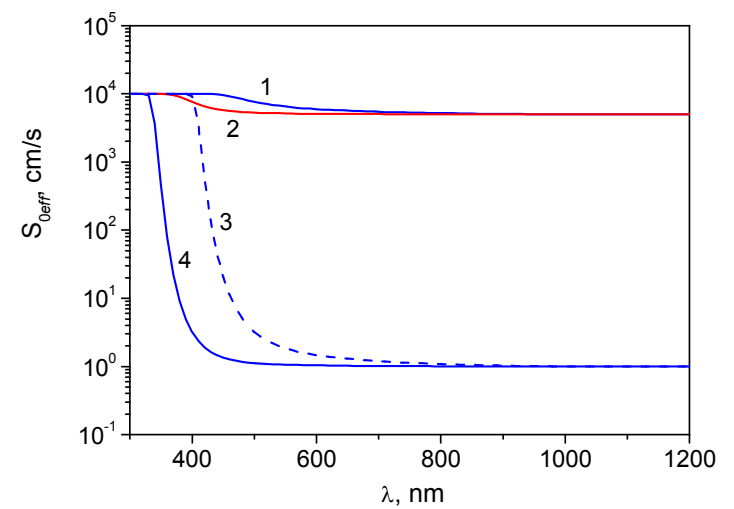

Fig. 5. Spectral dependences $S_{\text {eff }}(\lambda)$. Here, we used the following parameters: curve $1-S_{1}=10^{4} \mathrm{~cm} / \mathrm{s}, S_{2}=10^{4} \mathrm{~cm} / \mathrm{s}$, $d_{p}=10^{-4} \mathrm{~cm}$; curve $2-S_{1}=10^{4} \mathrm{~cm} / \mathrm{s}, S_{2}=10^{4} \mathrm{~cm} / \mathrm{s}, d_{p}=$ $10^{-5} \mathrm{~cm}$; curve $3-S_{1}=10^{4} \mathrm{~cm} / \mathrm{s}, S_{2}=1 \mathrm{~cm} / \mathrm{s}, d_{p}=10^{-4} \mathrm{~cm}$; curve $4-S_{1}=10^{4} \mathrm{~cm} / \mathrm{s}, S_{2}=1 \mathrm{~cm} / \mathrm{s}, d_{p}=10^{-5} \mathrm{~cm}$.

In the case when $\alpha d_{p}<1$, the value $\alpha^{-1}$ is the efficient thickness of the sub-surface layer. Taking it into account, one can transform (6) to the look

$$
S_{\text {eff }}(\lambda)=\frac{D_{1}}{L_{1}} \frac{S_{0} \frac{L_{1}}{D_{1}} \cosh \left(\frac{1}{\alpha(\lambda) L_{1}}\right)+\sinh \left(\frac{1}{\alpha(\lambda) L_{1}}\right)}{S_{0} \frac{L_{1}}{D_{1}} \sinh \left(\frac{1}{\alpha(\lambda) L_{1}}\right)+\cosh \left(\frac{1}{\alpha(\lambda) L_{1}}\right)} .
$$

As seen from (7), when $\alpha$ grows, i.e., the illumination wavelength $\lambda$ is decreased, in the case when $S_{0}>D_{1} / L_{1}$, the value $S_{e f f}(\lambda)$ tends to $S_{0}$, and limitations for the efficient speed of surface recombination due to diffusion bringing disappear. For intermediate $\lambda$ values, the values of the efficient speed of surface recombination tends to the value defined by (7) and the $S_{0}$ value.

Fig. 6 illustrates spectral dependences of the smallsignal photo-e.m.f. in the case of continuous illumination plotted using the expression from [2]

$$
\begin{aligned}
& \bar{V}_{p h}(\lambda)=\frac{k T}{q} \frac{\tilde{I}}{p_{0} \exp \left(\frac{q \bar{V}_{p h}}{k T}\right)} \frac{\alpha L}{\left(\alpha^{2} L^{2}-1\right)} \times \\
& \times\left[\left(S_{0} S_{d}+V_{d}^{2}\right) \sinh \left(\frac{d}{L}\right)+\left(S_{0}+S_{d}\right) V_{d} \cosh \left(\frac{d}{L}\right)\right]^{-1} \times \\
& \left\{\alpha L\left[\left(S_{d} \sinh \left(\frac{d}{L}\right)+V_{d} \cosh \left(\frac{d}{L}\right)\right)\left(1-R_{d} e^{-2 \alpha d}\right)-V_{d} e^{-\alpha d}\left(1-R_{d}\right)\right]\right. \\
& +S_{d} e^{-\alpha d}\left(1+R_{d}\right)- \\
& \left.-\left(S_{d} \cosh \left(\frac{d}{L}\right)+V_{d} \sinh \left(\frac{d}{L}\right)\right)\left(1+R_{d} e^{-2 \alpha d}\right)\right\},
\end{aligned}
$$

where $p_{0}$ is the concentration of minority charge carriers in the base, $\bar{V}_{p h}$ - e.m.f. of the open circuit under continuous illumination, $\widetilde{I}$ - intensity of modulated illumination, $V_{d}=D / L$ - speed of diffusion in the base.

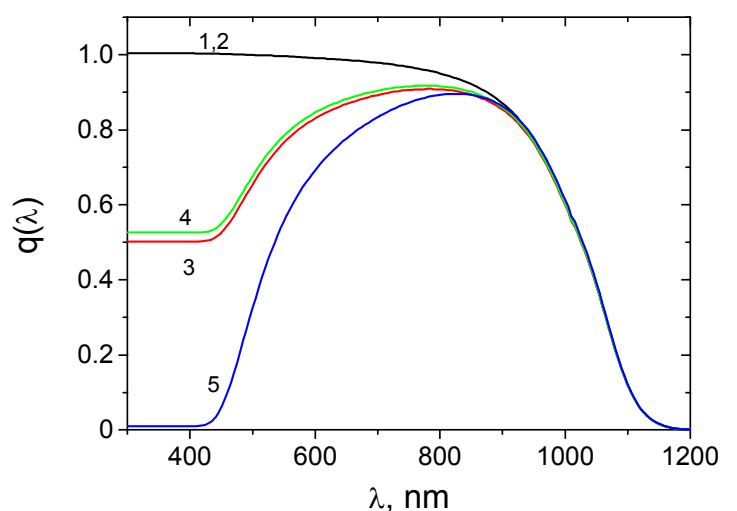

Fig. 6. Calculated and normalized spectral dependences of the small-signal photo-e.m.f. Used here are the following parameters: $L=200 \mu \mathrm{m}, \quad d=400 \mu \mathrm{m}, \quad S_{d}=10 \mathrm{~cm} / \mathrm{s}$, $R_{d}=1 \mathrm{~cm}, D=10 \mathrm{~cm}^{2} / \mathrm{s}$; the curve 1 is plotted for $S_{0}=$ const; curve $2-S_{1}=1 \mathrm{~cm} / \mathrm{s}, S_{2}=10^{4} \mathrm{~cm} / \mathrm{s}, d_{p}=0 ;$ curve $3-$ $S_{1}=10^{5} \mathrm{~cm} / \mathrm{s}, \quad S_{2}=10^{5} \mathrm{~cm} / \mathrm{s}, \quad d_{p}=10^{-4} \mathrm{~cm} ; \quad$ curve 4 $S_{1}=10^{4} \mathrm{~cm} / \mathrm{s}, \quad S_{2}=10^{4} \mathrm{~cm} / \mathrm{s}, \quad d_{p}=10^{-4} \mathrm{~cm} ; \quad$ curve 5 $S_{1}=10^{7} \mathrm{~cm} / \mathrm{s}, S_{2}=10^{5} \mathrm{~cm} / \mathrm{s}, d_{p}=10^{-4} \mathrm{~cm}$.

It is noteworthy that, for considering the theoretical results and comparing the experimentally obtained data, it seems purposeful to use the expression for the normalized photo-e.m.f. value:

$V\left(\lambda, \lambda_{m}\right)=\frac{V_{p h}(\lambda)}{V_{p h}\left(\lambda_{m}\right)}$,

where $\lambda_{m}$ is the illumination wavelength, for which the $V_{p h}(\lambda)$ value has its maximum.

If $S_{0 \text { eff }}=S_{0}=$ const, then the short-wave drop in $V_{p h}(\lambda)$ is absent independently of the $S_{0}$ value (see curves 1 and 2). If the dependence $S_{e f f}(\lambda)$ is described with the formula (5) when $S_{0 \text { eff }}(\lambda)>D / L$, then short-wave drop in $V_{p h}(\lambda)$ takes its place.

It should be noted that Exps (5) and (7) give the similar $S_{\text {eff }}(\lambda)$ dependences, and, for some definite values of parameters, practically coinciding are not only the dependences on the illumination wavelength $\lambda$, but boundary values in the long-wave and short-wave absorption ranges.

However, if one normalizes the values of the smallsignal photo-e.m.f. by its maximum value, then the spectral dependences $V_{p h}(\lambda)$ become ambiguous, and on conditions that $S_{0}>D_{1} / L_{1}$ they practically coincide (curves 3 and 4 ), if the ratio $S_{1} / S_{2}=$ const. Thus, to find $S_{1}$ and $S_{2}$ values, it is insufficient to use relative values and normalize by their maximum, as it is made when determining the diffusion length in bulk material, but it is important to measure absolute values of the photoe.m.f. $V_{p h}(\lambda)$.

At last, it should be noted that to realize the above approach it is necessary to provide validity of the criterion $w<d_{p}$, i.e., the width of SCR $w$ should be less than the thickness of the sub-surface layer with a decreased lifetime. In addition, using Exp. (3) for theoretical modeling, one can determine the values $D_{1}$ 
and $L_{1}$ by using comparison of experimental and theoretical values for the small-signal photo-e.m.f. within the range of the short-wave drop. Then from the expression $\tau_{1}=L_{1}^{2} / D_{1}$ one can find the value of efficient lifetime in the sub-surface layer of in the emitter of a solar cell.

\section{Spectral dependences of the internal quantum efficiency for the short-circuit current in solar cells with back metallization}

It should be noted that in the case of solar elements with back barriers and contact metallization (CETM), where minimization of the surface recombination rate on the illuminated surface is reached due to creation of an isotype $n^{+}-n$ junction (or $p-n$ junction), the layer with decreased values of lifetime and diffusion length for electron-hole pairs arises near the front surface. Therefore, it should be expected that in this case there can take place the dependences of the internal quantum efficiency for the short-circuit current, which decay with lowering the illumination wavelength. It is related with growth of the efficient speed of surface recombination in accord with the mechanism considered above. It is illustrated by depicted in Fig. 7 experimental spectral dependences for the internal quantum efficiency of solar cells with back metallization as well as by theoretical dependences calculated using the following expression

$$
\begin{aligned}
& q(\lambda)=\frac{\alpha(\lambda) L}{1-\alpha(\lambda)^{2} L^{2}} \\
& \left\{\frac{-\left[\frac{S_{e f f}(\lambda) L}{D}\left(1-e^{-\alpha d-d / L}\right)+\alpha L+e^{-\alpha d-d / L}\right]}{\cosh \left(\frac{d}{L}\right)+\frac{L}{D} S_{e f f}(\lambda) \sinh \left(\frac{d}{L}\right)}+\right. \\
& \left.+(1+\alpha L) e^{-\alpha d}\right\}
\end{aligned}
$$

and the formula (5).

Experimental dependences of the internal quantum efficiency have been measured using CETM samples with the structure $n^{+}-n-p^{+}\left(n^{+}\right)$and correspond to the following sequential processing: Q (rhombi, Fig. 7) initial CETM with a heavily doped $n+-$ layer and antireflection $\mathrm{SiO}_{2}$ coating; Qt1 (triangles) - the same CETM but after etching the anti-reflection $\mathrm{SiO}_{2}$ coating in HF solution; Qt2 (circles) - CETM with following thinning the $n+-$ layer in the etchant for silicon, and Qt3 (squares) - CETM with the fully etched $n+$ - layer (structure $\left.n-p^{+}\left(n^{+}\right)\right)$.

Theoretical dependences $q(\lambda)$ for the samples $\mathrm{Q}$ and Qt1 were plotted using the formulae (10) and (7), while for the samples Qt2 and Qt3 - the formulae (10) and (5). For the samples Q and Qt1, coincidence between theory and experiment was obtained at the same values of the diffusion coefficient and diffusion length in the heavily doped layer: $D_{1}=0.84 \mathrm{~cm}^{2} / \mathrm{s}$ and
$L_{1}=4.2 \mu \mathrm{m}$, as well as for the same value $S_{0} \approx 3 \cdot 10^{4} \mathrm{~cm} / \mathrm{s}$. In our opinion, it confirms that in this case the short-wave drop in $q(\lambda)$ is entirely related with Auger recombination in the isotype $n-n^{+}$- junction.

In the case of the samples Qt2 and Qt3, where the heavily doped layer was thinned (Qt2) or entirely etched (Qt3), comparison of the experimental dependence for the internal quantum efficiency with the theoretical one was made as in [8] with using the expression for the spectral dependence of the efficient speed of surface recombination (5). We believe that in the case of CETM Qt3 an exhausting band bend is realized on the silicon surface, and availability of the short-wave drop is related with a residual damaged layer. The value of the surface recombination speed $S_{1}$ is in our case $5 \cdot 10^{4} \mathrm{~cm} / \mathrm{s}$ for both samples, $S_{2}-2 \cdot 10^{3} \mathrm{~cm} / \mathrm{s}$ (Qt2) and $5 \cdot 10^{3} \mathrm{~cm} / \mathrm{s}$ (Qt3), while the thickness of the residual damaged layer is 0.35 and $0.3 \mu \mathrm{m}$, respectively for the samples Qt2 and Qt3, which correlates well with the results obtained in [8].

It should be noted that usage of the obtained values $D_{1}$ and $L_{1}$ enables to determine the lifetime in the isotype $n-n^{+}$junction. It is approximately equal to $2 \cdot 10^{-7} \mathrm{~s}$. These lifetime values are indicative of the fact that the level of doping within the $n^{+}$-range of the isotype junction is close to $10^{19} \mathrm{~cm}^{-3}$. This situation can be essentially improved, if the doping level in this layer will be reduced down to the value of $10^{18} \mathrm{~cm}^{-3}$. In this case, the spectral dependences for the internal quantum efficiency of short-circuit current normalized by the unity in the peak point have a look depicted in Fig. 7 (curves 5 and 6). When $S_{0}=5 \cdot 10^{2} \mathrm{~cm} / \mathrm{s}$, the drop in $q(\lambda)$ at $\lambda<900 \mathrm{~nm}$ is absent (curve 5), while for $S_{0}=2 \cdot 10^{2} \mathrm{~cm} / \mathrm{s}$, the dependence $q(\lambda)$ when $\lambda<900 \mathrm{~nm}$ has a growing character (curve 6 ).

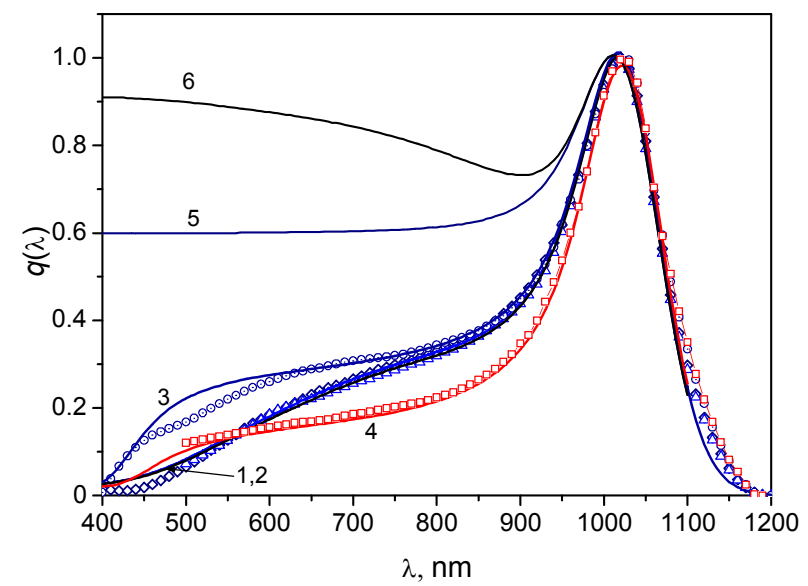

Fig. 7. Normalized spectral dependences for the internal quantum efficiency of silicon solar cells with back metallization. Dots - experimental dependences for various samples, lines - theoretical ones. The used parameters are as follows: $L=320 \mu \mathrm{m}, \quad d=380 \mu \mathrm{m}, R_{d}=0.4$; curve 1 $D_{1}=0.84 \mathrm{~cm}^{2} / \mathrm{s}, L_{1}=4.3 \mu \mathrm{m}, S_{0}=3 \cdot 10^{4} \mathrm{~cm} / \mathrm{s} ;$ curve 2 $D_{1}=0.84 \mathrm{~cm}^{2} / \mathrm{s}, L_{1}=4.3 \mu \mathrm{m}, S_{0}=3 \cdot 10^{4} \mathrm{~cm} / \mathrm{s}$, curve 3 $S_{1}=3 \cdot 10^{4} \mathrm{~cm} / \mathrm{s}, \quad S_{2}=2 \cdot 10^{3} \mathrm{~cm} / \mathrm{s}, \quad d_{p}=0.35 \mu \mathrm{m} ;$ curve 4 $S_{1}=3 \cdot 10^{4} \mathrm{~cm} / \mathrm{s}, \quad S_{2}=5 \cdot 10^{3} \mathrm{~cm} / \mathrm{s}, \quad d_{p}=0.3 \mu \mathrm{m} ;$ curve 5 $D_{1}=3 \mathrm{~cm}^{2} / \mathrm{s}, \quad L_{1}=50 \mu \mathrm{m}, \quad S_{0}=5 \cdot 10^{2} \mathrm{~cm} / \mathrm{s} ; \quad$ curve 6 $D_{1}=3 \mathrm{~cm}^{2} / \mathrm{s}, L_{1}=50 \mu \mathrm{m}, S_{0}=2 \cdot 10^{2} \mathrm{~cm} / \mathrm{s}$. 


\section{Conclusions}

As a result of our analysis concerning the mechanisms of the short-wave drop in the short-circuit current and small-signal photo-e.m.f. in silicon solar cells, we have ascertained:

1) short-wave drop of the short-circuit current in solar elements of standard design (with two-sided metallization) is related with the enhanced efficient speed of surface recombination on the illuminated surface. A high concentration of surface recombination centers and interband Auger recombination under high levels of doping the emitter layer can serve as mechanisms increasing the speed of surface recombination;

2) short-wave drop in spectral dependences of the small-signal photo-e.m.f. in solar cells of standard construction takes place due to availability of diffusion limitation for inflow of non-equilibrium carriers to recombination sites, which is related with a decreased lifetime in the emitter layer. This drop can be modeled both via the growing efficient speed of surface recombination $S_{\text {eff }}(\lambda)$ within the range of strong absorption, and with account of the decreased lifetime and diffusion coefficient of nonequilibrium charge carriers in the emitter layer;

3) short-wave drop of the short-circuit current in solar elements with back metallization has the same nature as that of the small-signal photo-e.m.f. in solar cells of standard construction and can be described by using the same parameters. The relationships obtained in our work enables to optimize the doping level in the isotype junction, which allows essential increase in the internal quantum efficiency for the short-circuit current in solar elements with back metallization in the shortwave absorption range;

4) it has been shown that investigations of spectral dependences within the short-wave range enable to obtain specific information upon changes of recombination parameters on surface and in bulk of photosensitive silicon with this non-destructive method.

In particular, juxtaposing the theory and experiment, one can determine parameters characterizing sub-surface properties of solar elements, namely: the thickness of the sub-surface layer with enhanced recombination, lifetime in it, as well as the $S_{\text {eff }}(\lambda)$ dependences.

\section{References}

1. Standard test methods minority carrier diffusion length in extrinsic semiconductors by measurement of steady-state surface photovoltage. Annual book of ASTM standards 10.05, F 391-96. - 1997. P. $150-158$.

2. A.P. Gorban, V.P. Kostylyov, A.V. Sachenko, A.A. Serba, V.V. Chernenko, Theoretical and experimental analysis of recombination parameters in high-efficient silicon solar cells // Optoelektronika $i$ poluprovodnikovaya tekhnika, 37, p. 61-68, 2002 (in Russian).

3. A.P. Gorban, V.P. Kostylyov, V.G. Litovchenko, A.V. Sachenko, O.V. Snitko, Peculiarities of spectral dependences for photo-current in surface-barrier structures based on silicon // Ukrainskii fizicheskii zhurnal, 34 (3), p. 404-407, 1989 (in Russian).

4. A.P. Gorban, A.V. Sachenko, V.P. Kostylyov and N.A. Prima // Semiconductor Physics, Quantum Electronics and Optoelectronics, 3 (2), P.322-329 (2000).

5. A.V. Sachenko, O.V. Snitko, Photoeffects in subsurface layers of semiconductors. Kyiv, "Naukova dumka", 1984, $231 \mathrm{p}$.

6. V.A. Zuyev, V.G. Popov, A.V. Sachenko, On anomalous short-wave drop in photo-effects // Fizika i tekhnika poluprovodnikov, 15 (3), P. 408 411, 1981 (in Russian).

7. A. Farenbruch, R. Bube. Solar elements: theory and experiment. Moscow, "Energoatomizdat", 1987 (in Russian).

8. A.V. Sachenko, V.P. Kostylyov, V.G. Litovchenko, V.G. Popov, B.M. Romanyuk, V.M. Naseka, T.V. Slusar, S.I. Kirillova, F.F. Komarov // Recombination characteristics of single-crystalline silicon wafers with a damaged near-surface layer //Ukrainian Journal of Physics, 58 (2), P. 142-150 (2013). 